\title{
The concurrent and longitudinal associations of temperament and nutritional risk factors in early childhood
}

M. van den Heuvel, Y. Chen, K. Abdullah, J. L. Maguire, P. C. Parkin, C. S. Birken, on behalf of the TARGet Kids! Collaboration

Version Post-print/accepted manuscript

Citation van den Heuvel, M., Chen, Y., Abdullah, K., Maguire, J. L., Parkin, P. (published version) C., Birken, C. S., and on behalf of the TARGet Kids! Collaboration (2017) The concurrent and longitudinal associations of temperament and nutritional risk factors in early childhood. Pediatric Obesity, 12: 431-438. doi: 10.1111/ijpo.12161.

Publisher's Statement This is the peer reviewed version of the following article: van den Heuvel, M., Chen, Y., Abdullah, K., Maguire, J. L., Parkin, P. C., Birken, C. S., and on behalf of the TARGet Kids! Collaboration (2017) The concurrent and longitudinal associations of temperament and nutritional risk factors in early childhood. Pediatric Obesity, 12: 431-438. doi: 10.1111/ijpo.12161, which has been published in final form at DOI: 10.1111/ijpo.12161. This article may be used for non-commercial purposes in accordance with Wiley Terms and Conditions for Self-Archiving.

How to cite TSpace items

Always cite the published version, so the author(s) will receive recognition through services that track citation counts, e.g. Scopus. If you need to cite the page number of the author manuscript from TSpace because you cannot access the published version, then cite the TSpace version in addition to the published version using the permanent URI (handle) found on the record page.

This article was made openly accessible by $U$ of 'T Faculty.

Please tell us how this access benefits you. Your story matters. 
The concurrent and longitudinal associations of temperament and nutritional risk

factors in early childhood

3

4

5

6

8

Meta van den Heuvel MD MSc ${ }^{1,4,5}$, Yang Chen MSc MA ${ }^{1,2}$, Kawsari Abdullah MBBS ${ }^{1,2}$, Jonathon L. Maguire MD MSc FRCPC ${ }^{1,2,3,4,5}$, Patricia C. Parkin MD FRCPC ${ }^{1,2,5}$ Catherine S. Birken MD MSc FRCPC ${ }^{1,2,3,5}$ on behalf of the TARGet Kids! Collaboration*

${ }^{*}$ TARGet Kids! Collaboration - Scientific Committee: Kawsari Abdullah, Laura N. Anderson, Catherine S. Birken, Cornelia M. Borkhoff, Sarah Carsley, Yang Chen, Mikael Katz-Lavigne, Kanthi Kavikondala, Christine Koroshegyi, Grace Jieun Lee, Jonathon L. Maguire, Dalah Mason, Jessica Omand, Patricia C. Parkin, Navindra Persaud, Meta van den Heuvel, Weeda Zabih; Site Investigators: Jillian Baker, Tony Barozzino, Joey Bonifacio, Douglas Campbell, Sohail Cheema, Brian Chisamore, Karoon Danayan, Paul Das, Mary Beth Derocher, Anh Do, Michael Dorey, Sloane Freeman, Keewai Fung, Charlie Guiang, Curtis Handford, Hailey Hatch, Sheila Jacobson, Tara Kiran, Holly Knowles, Bruce Kwok, Sheila Lakhoo, Margarita LamAntoniades, Eddy Lau, Fok-Han Leung, Jennifer Loo, Sarah Mahmoud, Rosemary Moodie, Julia Morinis, Sharon Naymark, Patricia Neelands, James Owen, Michael Peer, Marty Perlmutar, Navindra Persaud, Andrew Pinto, Michelle Porepa, Nasreen Ramji, Noor Ramji, Alana Rosenthal, Janet Saunderson, Rahul Saxena, Michael Sgro, Susan Shepherd, Barbara Smiltnieks, Carolyn Taylor, Thea Weisdors, Sheila Wijayasinghe, Peter Wong, Ethel Ying, Elizabeth Young.

\section{Affiliations:}

${ }^{1}$ Pediatric Outcomes Research Team (PORT), Division of Pediatric Medicine, Department of Pediatrics, The Hospital for Sick Children, Toronto, Ontario;

${ }^{2}$ Institute for Health Policy, Management and Evaluation, University of Toronto, Toronto, Ontario;

${ }^{3}$ The Applied Health Research Centre of the Li Ka Shing Knowledge Institute of St. Michael's Hospital, University of Toronto, Toronto, Ontario;

${ }^{4}$ Department of Pediatrics, St. Michael's Hospital, Toronto, Ontario; ${ }^{5}$ Department of Pediatrics, Faculty of Medicine, University of Toronto, Toronto, Ontario;

\section{Corresponding Author:}

Meta van den Heuvel, MD MSc

Social Pediatrician, Academic Pediatric Fellow

Hospital for Sick Children

555 University Ave, M5G 1X8, Toronto, ON, Canada

Located: Peter Gilgan Center for Research and Learning

10th floor, room: 10.9830

686 Bay Street, M5G0A4, Toronto, ON, Canada

Tel: 416-813-7654 ext. 301544; Fax: 416 813-5663

mathilda.vandenheuvel@sickkids.ca

Keywords: temperament, nutrition, early childhood 
1 Running title: temperament and nutrition in early childhood

3 What is already known about this subject

4 - Temperament is increasingly identified as an important early childhood

5 determinant of health

6 - High negative reactivity may be an early risk factor for childhood obesity

7 - Increased self-regulation might be protective for childhood obesity

9 What this study adds

10 - Three year old children with higher effortful control had reduced nutritional risk

11 factors and a reported healthier dietary intake at five years of age

12 - Higher negative affectivity was associated with a concurrent increased nutritional

13 risk but was not associated with a change in nutritional risk over time

14 - This study did not identify any relationship between surgency and nutritional risk

15 factors

16

17 Summary

18 Background: Early childhood temperament is increasingly recognized as an important

19 attribute that may impact screen time use, outdoor play and childhood obesity. The

20 relationship between temperament and nutrition in preschool children is less clear.

21

22 Objective: To investigate if temperament dimensions (negative affectivity, effortful control

23 and surgency) in early childhood are associated with nutritional risk factors. 
1 Methods: 678 children were followed (mean age at baseline visit 3.1 years; mean time to

2 follow-up 16.5 months). Parents reported on child temperament and nutritional risk

3 factors during regularly scheduled well-child clinic visits.

4 Results: A mixed effect model demonstrated a significant association between higher

5 negative affectivity $(1.03 ; 95 \% \mathrm{Cl} 0.69$ to 1.37$)$ and higher effortful control $(-0.88 ; 95 \% \mathrm{Cl}$

$6-1.27$ to -0.49 ) on concurrent nutritional risk, independent of covariates. Multivariate

7 linear regression analysis identified that higher effortful control, and not negative

8 affectivity, was significantly associated with a decrease in nutritional risk $(-0.67 ; 95 \% \mathrm{Cl}$ -

91.10 to -0.24$)$ over time, independent of covariates. There was no relationship identified

10 between surgency and nutritional risk.

12 Conclusion: Three year old children with higher effortful control had reduced nutritional

13 risk at 5 years of age. Future nutritional risk prevention strategies may benefit from

14 interventions to increase effortful control in early childhood.

\section{Introduction}

17 Nutrition plays an essential role in growth and development, academic success and in

18 the social- and emotional well-being of children $(1,2)$. Important examples of nutrition

19 related risk factors and health outcomes in young children include prolonged bottle use

20 contributing to iron deficiency and increased screen time and excessive intake of sugar

21 sweetened beverages contributing to obesity $(2,3)$. Nutritional risk has been defined as

22 "the presence of risk factors that can lead to impaired nutritional status" (4).

23 Many child-, family- and social factors and their complex interactions influence

24 child nutrition (5). Temperament has been defined as biologically based individual

25 differences in reactivity and self-regulation, influenced over time by heredity and

26 experience (6). Each child has 3 main reactive dispositions of temperament: 1) negative 
1 affectivity (e.g. sadness, discomfort), 2) effortful control (e.g. attention shifting, focusing,

2 inhibitory, activational control) 3) extraversion/ surgency (e.g. positive emotionality,

3 activity, impulsivity) (7).

4 A recent workshop conducted by the National Institute of Health identified the

5 need for studies that address how individual differences in early temperament contribute

6 to obesity (8). Emerging evidence suggests that high negative reactivity may be an early

7 risk factor for childhood obesity (9-12). Other studies also identified associations

8 between low effortful control, high negative affectivity and more television viewing and

9 reduced outdoor play in young children $(13,14)$. Additionally, a recent study by Leung et

10 al (2015) in low-income preschoolers revealed that high levels of surgency predicted

11 more food responsiveness and enjoyment of food leading to a higher concurrent Body

12 Mass Index (BMI) (15). Only one study investigated the relationship between

13 temperament and food-intake directly. They concluded that both internalizing (anxious,

14 dependent) and externalizing (hyperactive, aggressive) temperament in 18-month old

15 children were risk factors for consuming more sweet drinks and foods at 3 and 7 years

16 (16). In addition, somewhat contradictory to the study of Leung et al, this study also

17 reported that high surgent (active, sociable) 18-month old infants were more likely to

18 consume a healthy diet with higher intake of fruits and vegetables daily at 3 and 7 years.

19 However this study was limited by assessment of temperament at only one time point

20 (18 months) and used a non-validated measure of temperament (16).

21 The present study tested the hypothesis that in early childhood higher negative

22 affectivity and lower effortful control are associated with an increased nutritional risk and

23 an unhealthy dietary intake over time. Furthermore, because of this somewhat

24 contradictory evidence that children with high surgency were both at risk of high BMI,

25 and yet had a higher fruit and vegetable intake, our study aimed to add to the literature

26 and examined the impact of temperament on later dietary intake. 


\section{Methods}

3 Study Design and Setting

4 This was a longitudinal cohort study of children, aged 3-5 years, recruited during

5 scheduled well-child clinic visits from primary care practices participating in TARGet

6 Kids! (17). TARGet Kids! is a community-based primary care research network in

7 Toronto, Canada. The study protocol, including recruitment procedures, has recently

8 been published (17).

9 At each annually scheduled well-child clinic visit standardized questionnaires

10 were completed by parents and anthropometric measures, including height and weight,

11 were collected by research assistants using standardized measures and procedures

12 (17). Children were included if they had a minimum of two assessments of both

13 temperament and nutritional risk. Exclusion criteria included children with health

14 conditions affecting growth (e.g. cystic fibrosis), any acute or chronic conditions (other

15 than asthma and high functioning autism), children with severe developmental delay and

16 families who were unable to communicate in English. All data generated from TARGet

17 Kids! were entered into a web-based data management system (Medidata Rave $\AA$ ).

18 Ethics approval for the study was obtained from Research Ethics Boards of the Hospital

19 for Sick Children and St. Michael's Hospital, Toronto, Canada.

\section{Measures}

\section{$22 \quad$ Nutritional Risk}

23 The primary outcome was nutritional risk, as measured by the total score of the Nutrition

24 Screening Tool for Every Pre- schooler (NutriSTEP @) (1). The NutriSTEP ® was

25 developed for children ages 3-5 years of age, and has been validated in multicultural

26 Canadian children by registered dieticians using a detailed history and 3-day dietary 
1 recall (1). The NutriSTEP $\AA$ includes 17 questions about nutritional risk factors including

2 five domains; eating behaviors, dietary intake, parental concerns about food and activity

3 (including food security), screen time duration and the use of supplements. Each

4 question has 2-5 response options. Examples of questions include "My child is not

5 hungry at meal times because he /she drinks all day", "My child eats meals while

6 watching TV" (response options: always, most of the time, sometimes, rarely, never).

7 We calculated the Total NutriSTEP ${ }^{\circledR}$ score by aggregating the score of each

8 question (1). Higher total NutriSTEP $\AA$ scores represent more nutritional risk factors and

9 a greater nutritional risk (range of scores $0-68$ ) (1). We used the continuous Total

10 NutriSTEP® score as main outcome. For a secondary analysis, we dichotomized the

11 Total NutriSTEP $\AA$ score in two groups: high (Total NutriSTEP $\AA$ score $>20$ ) and low

12 (Total NutriSTEP $\AA$ score $\leq 20$ ). In the clinical setting, the above-mentioned cut points

13 are used; for children that have a Total NutriSTEP $\AA$ score $>20$ recommendations

14 should be made to change the child's eating and activity habits (1).

15 Unhealthy dietary intake was determined from the dietary intake domain score of

16 the NutriSTEP® which included 6 questions about the intake of vegetables, fruit, grain,

17 milk, meat or meat alternatives and 'fast food' (1). Higher scores of the dietary intake

18 domain represent an unhealthier dietary intake (range of scores 0-24) (1).

20 Child Temperament Measure

21 Temperament was measured using the parent reported very short form of the Child

22 Behavior Questionnaire (CBQ-VSF) for children 3 - 7 years (7). The CBQ-VSF includes

2336 items and provides a comprehensive assessment of temperamental behavior

24 patterns and has demonstrated satisfactory internal consistency, criterion validity and

25 longitudinal stability $(7,18)$. Parents were asked to rate their child on a 7 -point scale

26 ranging from 1 (extremely untrue of your child) to 7 (extremely true of your child). For 
1 each child, the VSF-CBQ produced a summary score of each of the three separate

2 temperament domains: Negative Affectivity, Effortful Control and Surgency. A child with

3 a high level of negative affectivity has typically a lowered mood, is angry, fearful and

4 very difficult to soothe (7). Children with a high level of effortful control have the capacity

5 to suppress inappropriate responses, have better self-regulation and can maintain focus

6 on task-related activities (7). Children with high surgency levels are impulsive, enjoy

7 situations with high stimulus intensity and do not show discomfort in social situations (7).

\section{Covariates}

10 Relevant parental and family characteristics were assessed with a standardized survey

11 instrument developed for TARGet Kids!, based on the Canadian Community Health

12 Survey (19). Neighborhood income was measured using median neighborhood income

13 from census data. The height and weight of children and their parents were measured

14 using established protocols, and BMI was standardized to z-score BMI (zBMls) using the

15 World Health Organization growth standards (20). Parent BMI refers to the BMI of the

16 parent who attended the clinic visit. In our study $85.5 \%$ was maternal BMI and $17.4 \%$

17 paternal $\mathrm{BMI}$ and in $91.3 \%$ the same parent attended all visits.

18 A number of child, parent and household characteristics may also contribute to

19 the relationship between temperament and nutritional risk. For example mothers with a

20 university education and older mothers have been shown to have more positive toddler

21 feeding practices (21). The following clinically important covariates, that we identified

22 from literature were included in all adjusted analysis models: child factors (age, sex, z-

$23 \mathrm{BMI}$ ), parent factors (maternal education, maternal age and parent $\mathrm{BMI}$ ) and household

24 factors (neighborhood income) $(2,16,21)$.

\section{Statistical Analysis}


1 We calculated means, standard deviations and percentages to characterize the study

2 population, each of the three domains of temperament (Negative Affectivity, Surgency,

3 Effortful Control and Surgency), nutritional risk (Total NutriSTEP® score) and unhealthy

4 dietary intake (dietary domain score NutriSTEP $\AA$ ) at the baseline clinic visit and at

5 follow-up visits.

6 For the cross-sectional analysis we tested the overall association between the 3

7 temperament domains and the Total NutriSTEP® score. The analysis included all

8 temperament domains and the Total NutriSTEP® score at all three clinic visits

9 simultaneously $(\mathrm{T} 1, \mathrm{~T} 2, \mathrm{~T} 3)$ adjusted for covariates above (Figure 1). We used a mixed

10 effect model to accommodate the correlation within subjects. We performed the same

11 analysis with the dietary intake domain score of the NutriSTEP ${ }^{\circledR}$ as an outcome. A

12 secondary cross-sectional analysis was performed using mixed effect logistic regression

13 analysis with the dichotomized Total NutriSTEP® score as outcome.

14 For the longitudinal analysis we used multivariate linear regression model with

15 correlated error structures to examine the effect of the three temperament domains on

16 changes in Total NutriSTEP® score over time accounting for within-subject level

17 correlations, while adjusting for the previous Total NutriSTEP® score and covariates.

18 The multivariate linear regression model used the change in Total NutriSTEP $₫$ score

19 (between preceding visits) as dependent variable and the three temperament domains at

20 the previous visit as main explanatory variables. For example, for the first follow-up visit

21 (T2) we used previous temperament and Total NutriSTEP® score from the baseline visit

22 (T1) (Figure 1). The same analysis was performed for the secondary outcome, using the

23 dietary intake domain score of the NutriSTEP ${ }^{\circledR}$.

24 In all models we tested for interactions between age and the three temperament

25 domains; we planned a-priori to perform a stratified analysis by age groups if any

26 significant interactions were identified. Additionally we hypothesized that the impact of 
1 negative affect could be different depending on the child's effortful control, and we tested

2 for this interaction in the longitudinal model. It is unclear if child z-BMI is a true

3 confounder of the relationship between temperament and nutritional risk, and therefore

4 we performed a sensitivity analysis without child z-BMI $(5,9)$. Figure 1 presents a

5 schematic representation of our statistical analysis. Data was analyzed using $R$ version

6 3.0.3. (22).

\section{Results}

9678 children met inclusion criteria. All children had at least two clinic visits and 135

10 children had three clinic visits. The mean follow-up time was 16.5 months. Child, parent

11 and household characteristics are presented in table 1.

13 Cross-sectional overall association between temperament and nutritional risk (see

\section{Figure 1)}

15 Mixed effect modeling demonstrated that higher negative affectivity was associated with

16 an increased Total NutriSTEP $\AA$ score adjusted for covariates (adjusted $B$ estimate 1.03;

$1795 \% \mathrm{Cl} 0.69$ to 1.37$)$. In contrast, higher effortful control was associated with a

18 decreased Total NutriSTEP® score (adjusted $B-0.88 ; 95 \% \mathrm{Cl}-1.27$ to -0.49 ). No

19 association was identified between surgency and the Total NutriSTEP $\AA$ score (Table 2).

20 The overall association between the temperament domains and unhealthy dietary intake

21 demonstrated similar, but smaller effects, for both higher negative affectivity (adjusted $B$

$220.31 ; 95 \%-0.12$ to 0.50 ) and higher effortful control (adjusted $B-0.66 ; 95 \%-0.80$ to -

$23 \quad 0.44)$ (Table 2).

24 Logistic regression analysis of the dichotomized measure of the Total

25 NutriSTEP® score identified that children with higher negative affectivity had an

26 increased odds of nutritional risk (high vs. low: OR $1.58 ; 95 \% \mathrm{Cl} 1.11$ to 2.27 ). In 
1 contrast, higher effortful control was associated with a reduced nutritional risk (high vs.

2 low: OR $0.34 ; 95 \% \mathrm{Cl} 0.22$ to 0.53 ). No association between surgency and nutritional risk

3 was identified.

4 Longitudinal effect of the three early temperament domains on changes in nutritional risk

5 and unhealthy dietary intake (see Figure 1)

6 Table 2 presents the multivariate linear association between temperament and the

7 change in nutritional risk at follow-up, adjusted for the previous Total NutriSTEP $\AA$ score.

8 Each one point higher effortful control at the previous clinic visit was associated with a

9 significant reduction in the Total NutriSTEP $\AA$ score (adjusted $B-0.67 ; 95 \% \mathrm{Cl}-1.06$ to -

$10 \quad 0.28)$ at follow-up visits, independent of covariates. No significant associations were

11 observed between either negative affectivity or surgency and a change in Total

12 NutriSTEP $\AA^{\circledR}$ score over time.

13 A similar result was demonstrated when examining the change in unhealthy

14 dietary intake (Table 2). Each one point higher effortful control at the previous visit was

15 associated with a healthier dietary intake (reduced NutriSTEP ${ }^{\circledR}$ dietary intake domain

16 score) at follow up (adjusted $B-0.53 ; 95 \% \mathrm{Cl}-0.79$ to -0.26 ), independent of covariates.

17 No longitudinal relationship was identified between either negative affectivity or surgency

18 and a change in unhealthy dietary intake.

19 No interactions between age and temperament were identified (data not shown).

20 No interactions between efforfful control and negative affect were identified (data not

21 shown). No differences in results were identified when performing the analysis without

22 child $\mathrm{z}$-BMI (data available upon request).

\section{Discussion}

25 This study identified both a concurrent and longitudinal protective effect of higher

26 effortful control on nutritional risk and dietary intake in early childhood. Children at age 3 
1 who had higher effortful control had reduced nutritional risk and a reported healthier

2 dietary intake at age 5. Children with higher negative affectivity had a 1.6 times higher

3 odds and children with higher effortful control a 0.3 times reduced odds of concurrent

4 nutritional risk. This is the first study that examined temperament and nutritional risk

5 factors longitudinally in early childhood. We used data from a large prospective cohort of

6 children recruited from primary care settings and adjusted for important potential

7 confounders.

8 Contrary to our hypothesis, higher negative affectivity was not associated with a

9 change in nutritional risk over time. A mixed picture of the relation between greater

10 negative affectivity and weight related outcomes of children were also reported in a

11 recent review (9). One explanation could be that negative affectivity in preschool children

12 has been associated with both emotional overeating as well as food avoidant eating

13 behaviors such as food fussiness and less enjoyment of food, which can lead to mixed

14 effects on nutritional risk over time (23). Another explanation for finding only a

15 concurrent effect of negative affectivity could be differences in parenting style. In the

16 study of Leung et al, low-income preschoolers who experienced emotion regulation

17 difficulties were more likely to have tantrums over being denied food, but less likely to

18 eat in the absence of hunger (24). In other words, the negative behavior itself may not

19 be problematic for developing obesity; rather the caregivers' responses might be (9).

20 There are several possible mechanisms that might explain the concurrent and

21 longitudinal association between higher effortful control and lower nutritional risk and

22 healthier dietary intake. Firstly, children with higher effortful control may have a better

23 ability to focus their attention on their own satiety responses, which could result in less

24 concurrent excessive dietary intake (24). Secondly, children with higher effortful control

25 may be able to better redirect their emotions and behaviors. This may result in less

26 parental use of food or screen time over time to soothe their children $(24,25)$. Finally, in 
1 adults it has been demonstrated that stress can lead to a shift in preference from healthy

2 foods to foods with high-added sugar and fats (26). Children with higher effortful control

3 may have an improved ability to regulate their emotions and can better cope with stress

4 over time, which could result in a lower preference for an unhealthy dietary intake

$5(27,28)$.

6 This study did not identify a concurrent or longitudinal relationship between surgency

7 and nutritional risk factors and dietary intake. Children with high surgency have a high

8 approach motivation and they may therefore learn more quickly to enjoy fruits and

9 vegetables (16). In contrast Leung et al identified that low-income preschoolers with

10 higher surgency were more inclined to overeat in response to external cues and to eat in

11 the absence of hunger $(15,24)$. Surgency could also shape parenting behavior; parents

12 may feed children with more surgent behavior more often (16). In our study, the

13 relationship between surgency and nutritional risk factors could be absent because of

14 differences in parenting behavior and possibly because of the inclusion of both the

15 eating of fruits and vegetables and eating of 'fast food' in the dietary intake NutriSTEP $\AA$

16 subscore.

17 Limitations of our study include the lack of measurement of parenting style.

18 Parenting style could have a mediating influence on the development of temperament

19 and nutritional risk factors in preschool children (9). Not all children had three clinic

20 visits, however there were no significant differences between the two groups, except for

21 zBMI for which we adjusted our analysis. Also, the parent that attended the clinic visit

22 was not always the same parent. However both maternal and paternal BMI are

23 established as independent risk factors for obesity in young children (29) .

24 Furthermore our study relied on parent report of temperament and nutritional risk,

25 which could have lead to ascertainment bias. Our study was conducted with a large

26 group of children in primary care offices, and therefore direct observation was not 
1 feasible. However, both the NutriSTEP $\AA$ and the CBQ-VSF have demonstrated strong

2 measurement properties when validated against direct observation $(1,7)$. Although there

3 is theoretical support to investigate the relationship between temperament and nutritional

4 risk (including dietary intake), there was no theoretical base for a relationship between

5 temperament and food security.. Our study may have also been limited by a relatively

6 low nutritional risk profile, and our demographic data revealed high parental education.

7 The results may therefore not be generalizable to other populations.

8 Results from this study contribute to the growing recognition of temperament as

9 an important early childhood behavioral risk factor in child nutritional outcomes. This

10 study used a longitudinal design and although causation cannot be directly inferred, it

11 represents a stronger design than previous studies. Results from this study suggests

12 that early childhood temperament impacts later nutritional risk providing evidence that

13 preventive strategies for decreasing nutritional risk factors in preschool children might

14 consider approaches to address temperament. While temperament of individuals tend to

15 be relatively stable over time, there is evidence that links between early temperament

16 and child outcomes are malleable and that self-regulation can be improved with

17 interventions promoting sensitive, responsive parenting (9). Future research should

18 explore parenting style as a mediating factor in relationship between temperament and

19 nutritional risk in preschool children.

20 In summary, this study demonstrated a concurrent and longitudinal protective effect

21 of high effortful control on nutritional risk factors in preschool children. New nutritional

22 risk prevention strategies might consider programming to increase effortful control as

23 opposed to decreasing negative affectivity in preschool children $(9,30)$.

\section{Conflict of Interest Statement}

26 All authors declare that they have no competing interests 


\section{Acknowledgements}

$3 \mathrm{MH}$ and $\mathrm{CB}$ were involved in the study design. All authors critically reviewed the study

4 design. $\mathrm{YC}$ analyzed all data. $\mathrm{MH}$ and $\mathrm{CB}$ wrote the first draft of the manuscript and

5 each author listed has revised and approved the submission of this manuscript and

6 takes full responsibility for the manuscript.

7 This work was supported by CIHR, SickKids Foundation, SMH foundation, Physician

8 Services Incorporated. Funding agencies had no role in the design and conduct of the

9 study, collection, management, analyses or interpretation of the results of this study or in

10 the preparation, review, or approval of the manuscript.

11 We would like to thank Dr. S.A. Reijneveld for critically reviewing the manuscript.

12 Additionally, the authors thank all participating families for their time and involvement in

13 TARGet Kids! and are grateful to all practitioners who are currently involved in the

14 TARGet Kids! research network. Steering Committee: Tony Barozzino, Brian

15 Chisamore, Mark Feldman, Moshe Ipp. Research Team: Kathleen Abreo, Tarandeep

16 Malhi, Antonietta Pugliese, Megan Smith, Laurie Thompson. Applied Health Research

17 Centre: Gerald Lebovic, Magda Melo, Patricia Nguyen. Mount Sinai Services Laboratory:

18 Azar Azad.

21 References

22 1. Randall Simpson JA, Keller HH, Rysdale LA, Beyers JE. Nutrition Screening Tool for

23 Every Preschooler (NutriSTEP): validation and test-retest reliability of a parent-

24 administered questionnaire assessing nutrition risk of preschoolers. Eur $\mathrm{J}$ Clin Nutr

25 2008; 62: 770-780. 
1 2. Ogata BN, Hayes D. Position of the Academy of Nutrition and Dietetics: nutrition 2 guidance for healthy children ages 2 to 11 years. J Acad Nutr Diet 2014; 114: 125731276.

4 3. Sutcliffe TL, Khambalia A, Westergard S, Jacobson S, Peer M, Parkin PC. Iron 5 depletion is associated with daytime bottle-feeding in the second and third years of life.

6 Arch Pediatr Adolesc Med 2006; 160: 1114-1120.

7 4. Identifying patients at risk: ADA's definitions for nutrition screening and nutrition 8 assessment. Council on Practice (COP) Quality Management Committee. J Am Diet 9 Assoc 1994; 94: 838-839.

10 5. Bergmeier H, Skouteris H, Horwood S, Hooley M, Richardson B. Associations 11 between child temperament, maternal feeding practices and child body mass index 12 during the preschool years: a systematic review of the literature. Obes Rev 2014; 15: 91318.

14 6. Rothbart M, Derryberry D. Development of individual differences in temperament. In:

15 Hillsdale N, Lawrence Erlbaum Associates I (eds) Advances in developmental

16 psychology. 1981, pp. 37.

17 7. Putnam SP, Rothbart MK. Development of short and very short forms of the Children's

18 Behavior Questionnaire. J Pers Assess 2006; 87: 102-112.

19 8. Lumeng JC, Taveras EM, Birch L, Yanovski SZ. Prevention of obesity in infancy and 20 early childhood: a national institutes of health workshop. JAMA Pediatr 2015; 169: 48421490.

22 9. Anzman-Frasca S, Stifter CA, Birch LL. Temperament and childhood obesity risk: a 23 review of the literature. J Dev Behav Pediatr 2012; 33: 732-745.

24 10. Faith MS, Hittner JB. Infant temperament and eating style predict change in 25 standardized weight status and obesity risk at 6 years of age. Int J Obes (Lond) 2010;

26 34: 1515-1523.

27 11. Slining MM, Adair L, Goldman BD, Borja J, Bentley M. Infant temperament 28 contributes to early infant growth: A prospective cohort of African American infants. Int $J$ 29 Behav Nutr Phys Act 2009; 6: 51-5868-6-51.

30 12. Francis $L A$, Susman EJ. Self-regulation and rapid weight gain in children from age 3 31 to 12 years. Arch Pediatr Adolesc Med 2009; 163: 297-302.

32 13. Radesky JS, Silverstein M, Zuckerman B, Christakis DA. Infant Self-Regulation and

33 Early Childhood Media Exposure. Pediatrics 2014;133: e1172-8.

34 14. Julia R. Sharp, Jonathon Maguire, Sarah Carsley, et al. Temperament is associated

35 with free play in young children. PAS Meeting, 2014, reference nr: 3803105. 
1 15. Leung CY, Miller AL, Kaciroti NA, Chen YP, Rosenblum K, Lumeng JC. Low-income 2 pre-schoolers with higher temperamental surgency enjoy and respond more to food,

3 mediating the path to higher body mass index. Pediatr Obes 2015;

4 doi:10.1111/ijpo.12042.

5 16. Vollrath ME, Stene-Larsen K, Tonstad S, Rothbart MK, Hampson SE. Associations 6 between temperament at age 1.5 years and obesogenic diet at ages 3 and 7 years. $J$

7 Dev Behav Pediatr 2012; 33: 721-727.

8 17. Carsley S, Borkhoff CM, Maguire JL, et al. Cohort Profile: The Applied Research

9 Group for Kids (TARGet Kids!). Int J Epidemiol 2014; doi: 10.1093/ije/dyu123.

10 18. Putnam SP, Gartstein MA, Rothbart MK. Measurement of fine-grained aspects of

11 toddler temperament: the early childhood behavior questionnaire. Infant Behav Dev

12 2006; 29: 386-401.

13 19. StatsCan. Canadian Health Measures Survey. Detailed information for January 2012 14 to December 2013 (Cycle 3). [WWW Document] URL

15 http://www23.statcan.gc.ca/imdb/p2SV.pl?Function=getSurvey\&SDDS=5071 (accessed

16 November 2015).

17 20. de Onis M, Onyango A, Borghi E, et al. Worldwide implementation of the WHO Child

18 Growth Standards. Public Health Nutr 2012; 15: 1603-1610.

19 21. Hendricks K, Briefel R, Novak T, Ziegler P. Maternal and child characteristics

20 associated with infant and toddler feeding practices. J Am Diet Assoc 2006; 106: S135-

2148 .

22 22. $\mathrm{R}$ Core Team. $\mathrm{R}$ Core Team. R: A language and environment for statistical

23 computing. R Foundation for Statistical computing. Vienna, Austria. 2014; R version

$24 \quad 3.0 .3$.

25 23. Haycraft E, Farrow C, Meyer C, Powell F, Blissett J. Relationships between

26 temperament and eating behaviours in young children. Appetite 2011; 56: 689-692.

27 24. Leung CY, Lumeng JC, Kaciroti NA, Chen YP, Rosenblum K, Miller AL. Surgency

28 and negative affectivity, but not effortful control, are uniquely associated with obesogenic

29 eating behaviors among low-income preschoolers. Appetite 2014; 78: 139-146.

30 25. Thompson AL, Adair LS, Bentley ME. Maternal characteristics and perception of

31 temperament associated with infant TV exposure. Pediatrics 2013; 131: e390-7.

32 26. Zellner DA, Loaiza S, Gonzalez Z, et al. Food selection changes under stress.

33 Physiol Behav 2006; 87: 789-793.

34 27. Mayer SE, Abelson JL, Lopez-Duran NL. Effortful control and context interact in

35 shaping neuroendocrine stress responses during childhood. Horm Behav 2014; 66: 457-

36465. 
1 28. Miller AL, Horodynski MA, Herb HE, et al. Enhancing self-regulation as a strategy for

2 obesity prevention in Head Start preschoolers: the growing healthy study. BMC Public

3 Health 2012; 12: 1040-2458-12-1040.

4 29. Morandi A, Meyre D, Lobbens S, et al. Estimation of newborn risk for child or

5 adolescent obesity: lessons from longitudinal birth cohorts. PLoS One 2012; 7: e49919.

6 30. Rollins BY, Savage JS, Fisher JO, Birch LL. Alternatives to restrictive feeding

7 practices to promote self-regulation in childhood: a developmental perspective. Pediatr

8 Obes 2015;doi:10.1111/ijpo.12071. 
$4 \quad$ Table 1 Baseline characteristics

\begin{tabular}{|llll|}
\hline Child characteristics & All children & $\begin{array}{l}\text { Children with 2 } \\
\text { clinic visits }\end{array}$ & $\begin{array}{l}\text { Children with } 3 \\
\text { clinic visits }\end{array}$ \\
Number of children & 678 & 543 & 135 \\
Boys (n,\%) & $336(49.6)$ & $270(49.7)$ & $66(48.9)$ \\
Age, months (SD) & $38.1 \pm 2.3$ & $37.7 \pm 2.9$ & $37.3 \pm 1.9$ \\
z-BMI (SD) (kg/m2) & $0.27 \pm 1.0$ & $0.22 \pm 0.98$ & $0.48 \pm 1.0$ \\
Negative Affectivity & $3.7 \pm 0.8$ & $3.7 \pm 0.8$ & $3.6 \pm 0.8$ \\
Effortful Control & $5.4 \pm 0.7$ & $5.4 \pm 0.7$ & $5.4 \pm 0.7$ \\
Surgency & $4.5 \pm 0.8$ & $4.5 \pm 0.8$ & $4.6 \pm 0.8$ \\
Total NutriSTEP @ score & $13.7 \pm 6.2^{3}$ & $13.9 \pm 6.4$ & $13.1 \pm 5.3$ \\
Dietary Intake domain score & $6.7 \pm 3.2^{4}$ & $6.7 \pm 3.2$ & $6.7 \pm 3.0$ \\
& & & \\
Parent \& Household characteristics & $36.1 \pm 4.4$ & $33.0 \pm 4.5$ & $32.7 \pm 3.7$ \\
Age of mother, years (SD) & $25.0 \pm 4.5$ & $25.0 \pm 4.4$ & $24.8 \pm 4.9$ \\
Parent BMl (SD) (kg/m2),2 & $63.6 \pm 30.5$ & $62.8 \pm 30.9$ & $66.3 \pm 28.9$ \\
Neighborhood income (SD) (1000 CAD) & & & \\
Maternal education (n, \%) & & $4(0.8)$ & 0 \\
Public school & $4(0.6)$ & $32(6.1)$ & $8(6.2)$ \\
High school & $40(5.9)$ & $493(93.2)$ & $122(93.9)$ \\
College / University & $615(90.7)$ & & \\
\hline
\end{tabular}

$6 \quad{ }^{1}$ Using WHO Child Growth Standards (20)

$7 \quad 2$ Parent BMI refers to the BMI of the parent who attended the clinic visit.

$8{ }^{3}$ Percentiles of the Total NutriSTEP ® score at baseline: 5th percentile 5; 25th percentile 9, 75th

9 percentile 17,95 th percentile 25

104 Percentiles of the Dietary Intake domain score at baseline: 5 th percentile 2; 25 th percentile 4;

11 75th percentile 9, 95th percentile 12

12 Abbreviations: $\mathrm{BMI}=$ Body Mass Index, $\mathrm{CAD}=$ Canadian Dollar. Missing data $\mathrm{z}-\mathrm{BMI} \mathrm{n}=8$, age of

13 mother $n=39$, parent BMI $n=82$, neighborhood income $n=56$, maternal education $n=17$ 
3 Figure 1 Schematic representation of statistical analysis

4

5 Legend:

6 A: Cross-sectional mixed effect model to test the overall associations of temperament on

7 nutritional risk at all three time points simultaneously.

8 B: Longitudinal multivariate linear regression model to test the effect of the previous

9 temperament domains on changes in nutritional risk (Total NutriSTEP® score) at follow-

10 up visits

11

12 T1 = baseline well-child clinic visit, T2 = follow-up well-child clinic visit, T3 = follow-up

13 well-child clinic visit

14

15

16

17

18

19

20

21

22

23

24

25 